\title{
Le Commerce de Proximité de la Grande Distribution en Tunisie: Cas de la Chaîne Aziza
}

\author{
Sami Ben Fguira ${ }^{1}$, Mongi Belarem ${ }^{2}$ \\ ${ }^{1}$ SYFACTE laboratory, University of Sfax- Tunisia \\ ${ }^{2}$ Department of Geography and Geographic Information System, Faculty of Arts and Humanities, King \\ Abdulaziz University, Jeddah, KSA.SYFACTE laboratory, University of Sfax-ATCIG - Tunisia
}

*Corresponding Author: Mongi Belarem, Department of Geography and Geographic Information Systems, Faculty of Arts and Humanities, King Abdulaziz University, Jeddah, KSA. SYFACTE laboratory, University of Sfax- ATCIG - Tunisia

\begin{abstract}
In recent years, mass retailing has undergone an important and rapid evolution, despite a generally unstable political and economic framework. We were witnessing an evolution and remodeling of the large retailers. In fact, large-scale retailers continue to win new shares in the retail market. In addition, it knows the introduction of new forms (Hard discount) and targeted new locations (local trade). This new strategy is adopted by "Aziza", a new hard-discount local food retailer, which appeared in 2014. It belongs to the Slama group active in the agri-food sector via GIAS brands (Goldina, Nejma), Ice Cream (Nassim), Hightech $(\mathrm{MB})$. The strategy of the new chain is to locate near the most populated areas including the popular neighborhoods. This brand now has more than 170 stores spread mainly in large and medium-sized cities.
\end{abstract}

Keywords: Aziza, hard discount, proximity, supermarket

\section{INTRODUCTION}

En Tunisie (carte 1), l'émergence du secteurde la grande distribution a été favorisée par une forte demande locale et un accroissement du pouvoir d'achat de la population. En effet, l'essor de la grande distribution et la prolifération des magasins dans l'espace urbain tunisien trouve son origine dans l'évolution du niveaudevie et de la consommation des citadinssuite à l'améliorationde leursrevenus.Ainsi, le pouvoir d'achat du tunisien a augmenté de 5000 dinars tunisiensen 2009 à 7000 en 2015.Durant les deux dernières décennies, le paysage de la grande distribution moderne a connu, de profondes mutations. La grande distribution, a réussi à gagner de nouveaux parts des marchés du commerce de détail. D'après une étude de Mac SA publiée en $2017^{1}$, le secteur capteenviron $24 \%$ de la somme de vente du commerce de détail. Un deuxième changement notoire observé ces dernières années dans la structure générale de l'offre de la grande distribution moderneconcernel'arrivée d'une nouvelle chaîne spécialisée dans le discount alimentaire « Aziza ».

Le présent article se propose de saisir les principales mutations de la grande distribution en Tunisie particulièrement l'arrivée du hard discount sous l'enseigne «Aziza » ces dernières années.Partant des données et des travaux précédents sur le sujet, la première partiede ce travailest consacré à dresserun bilan du développement général du secteurde la grande distribution en Tunisie. Une deuxième partie, sera consacréeà explorerla nouvelle formule «hard discount » déployée par la chaîne «Aziza »en Tunisie, età examinerses techniques de différenciationpar rapport aux autres formes de la grande distribution. Enfin, nous nous intéressons, à travers une approche cartographique, à décrypter la stratégie d'expansion de cette enseigne,qui repose en particulier sur l'investissement de la proximité, à l'échelle nationale mais aussi plus locale.

\section{L'EVOLUTION DE LA GRANDE DISTRIBUTION EN TUNISIE}

\subsection{Historique de la Grande Distribution Moderne en Tunisie}

L'histoire de la grande distribution moderne en Tunisie est scandée par quatre phases aux caractéristiques bien distinctes :une phase pionnière qui remonte au début du $\mathrm{XX}^{\text {ème }}$ siècle.L'irruption

\footnotetext{
${ }^{1}$ www.masca.com.tn/masca-new/index.xhtml
} 
dans le paysage des villes tunisiennes des grandes surfaces de distribution a débuté en 1920 avec la création du « Magasin Général » à Tunis.Après dix ans, « Monoprix », ainstallé3 surfaces de vente ; uneà Tunis, une deuxième à Sfax et une autre à Sousse (Bennasr A., Azouazi Th., 2010). Cette première génération de magasins visait principalement une clientèle aisée et urbaine dela population coloniale et tunisienne.

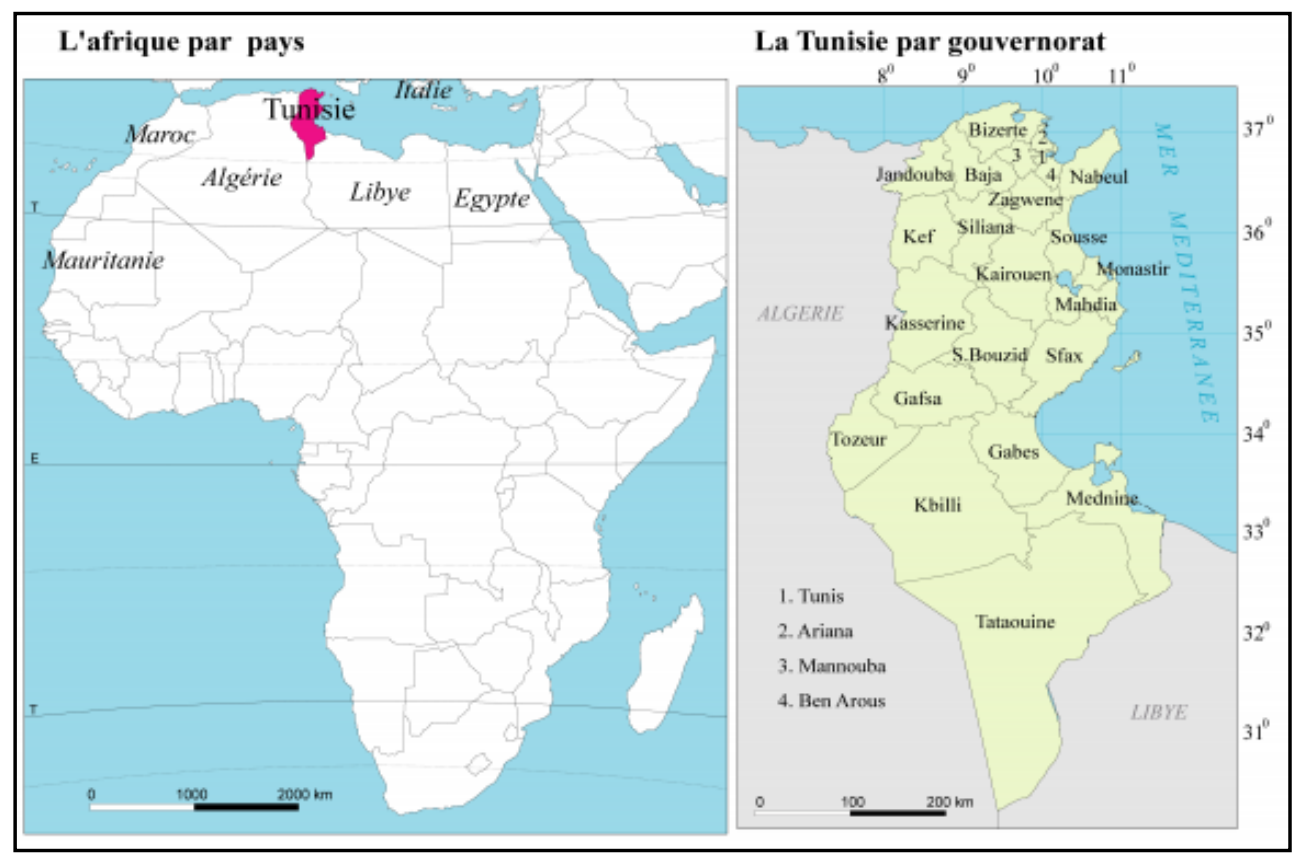

Carte1. Localisation de la zone d'étude (Tunisie) et son découpage par gouvernorat

Source : Ben Fguira et Belarem, 2018

Une deuxième phase de développement de la grande distribution s'est déclenchée dans la deuxième moitié des années 1990.Elle a été impulséepar la montée en puissanced'acteurs locaux qui ont multiplié les implantations des magasins notamment dans les grandes villes (le Grand Tunis, Sfax et le Sahel). Il s'agit principalement de l'enseigne semi-grossistes «PROMOGROS, d'une surface de vente de 5000mètres carrés) et l'enseigne BONPRIX ${ }^{2}$ qui a dominé le secteur de la grande distribution en Tunisie avec 44 magasins en $2005 »$ (Bennasr A., 2005).

Dès 2001, le paysage tunisiende la grande distribution est bouleversé : le pays accueille le premier hypermarché sous l'enseigne de la chaîne multinationale «Carrefour ». Trop vite, «Carrefour » est parvenu à devenir le leader de la grande distribution en Tunisie. En 2002, "Carrefour » a accaparépresquela moitié du chiffre d'affaires du secteur de la grande distribution (Bennasr A., Azouazi Th., 2010). Quatre ans plus tard, un deuxième hypermarché a ouvert ses portes (Géant ${ }^{3}$, $12000 \mathrm{~m}^{2}$ ) par l'entreprise internationale MEDIS.

A l'instar de nombreux pays en développement, en Tunisie, la modernisation du système de distribution a été portée par la classe aisée puis diffusée par la couchemoyenne (Amine A., 2012). «L'apparition de la grande distribution a été favorisée par unemodificationdu comportement du tunisiens déduit de plus en plus par le modèlede consommation européen. Ce changement du modèle de consommation est, dans un premier temps, apparu chez la classe aisée puis s'est étendu à la classe moyenne » (https://www.troyes.cci.fr/).Face à l'évolution de la consommation et des comportements d'achat, la grande distribution tunisienne a connu un développement considérable. Elle est parvenue à s'implanter durablement dans le marché du commerce de détail et s'étendre. «La part de marché de la grande distribution est passée de 5\% en 2000 à $12 \%$ en 2004 puis à $15 \%$ en 2008 »(Bennasr A., Azouazi Th., 2010).

\footnotetext{
${ }^{2}$ A l'origine filiale du groupe BATAM, la société BONPRIX, Bonprix est une enseigne et société de grande distribution constituée en 1998, est rachetée en avril 2006 par le groupe UTIC.

${ }^{3}$ GEANT est une société sœur de Monoprix.
} 
Depuis le déclenchement de la révolution tunisienne en 2011, la grande distribution a subi les retombées de la crise économique qu'a connue le pays. Le secteur est confronté à nouveaux défis émanant de la dégradation du pouvoir d'achat conjuguée à la hausse du taux d'inflation. Pour contourner et retarder cette crise économique, les enseignes du secteur s'efforcent à adapter de nouvelles stratégies et diversifier leurs offres. A cet effet, cesdernières années un nouveau format de distribution vient s'intégrer au paysage commercial : le hard discount. Ce dernier semble plus adapté aux attentes et exigences d'un consommateur à faible pouvoir d'achat.

\subsection{La Concurrence Entre les Enseignes}

Avec le lancement du premier hypermarché en 2001, la grande distribution a connu une importanterévolution. Dès lors, on a assisté à une accélération des installations des grandes et moyennes surfaces. D'après les chiffres, le parc de magasins de la grande distribution a évolué de 136 surfaces en 2005 à 447 en 2017 enregistrant un taux de croissance de l'ordre de 229\%. En effet, la part de la grande distribution augmente progressivement, danstransactions financières du commerce de commerce de détails, au détriment de la distribution traditionnelle. Durant la période 2007-2012, sa part a augmenté de $16 \%$ à environ $20 \%$, alors que la contribution traditionnelle s'est vurégresserde $84 \%$ à $80 \%$.

Aujourd'hui, «la grande distribution est principalement représentée par quatre grands acteurs »(https://docplayer.fr): Magasin général, Monoprix, Carrefour et Aziza (tableau 1).«En addition aux enseignes citées, d'autres petits acteurs agissent sur le marché de la grande distribution mais ne détiennent qu'une faible part de marché » (https://docplayer.fr). On peut à titre d'exemple citer : Afar (une enseigne à Kairouan) et Masmoudi (une enseigne à Sfax).

Tableau1. Les principales enseignes de la grande distribution en Tunisie

\begin{tabular}{|l|c|c|c|}
\hline & Date de création & Nombre de points de vente & Pourcentage \\
\hline Magasin général & 1883 & 91 & $20.36 \%$ \\
\hline Monoprix & 1933 & 87 & $19.46 \%$ \\
\hline Carrefour & 2001 & 92 & $20.58 \%$ \\
\hline Aziza & 2014 & 177 & $39.6 \%$ \\
\hline Total & & 447 & $100 \%$ \\
\hline
\end{tabular}

Source : sites web carrefour, Monoprix, Aziza, Magasin général

En effet, ces quatre groupes d'enseignes ont commencé à se développer des stratégiesdifférentes, pour monopoliserd'importantes parts de marché. Le développement des grands groupes de distribution en Tunisie s'est effectuéà la fois par des réorganisations internes et par une croissance externe.Ainsi, des opérations de fusions et acquisitions ont contribué à la construction des principaux groupes de distribution et à l'affirmationde leur avantage concurrentiel au détriment des autres entreprises de distribution indépendantes isolées.Parmi les opérations d'acquisitions les plus marquanteson peut citer le rachat des 43 magasins Bonprix par le groupe Chaibipropriétaire de Carrefour en septembre $2006 q u i$ lui a permis d'élargir son réseau de points de vente et d'augmenter son chiffre d'affaires.Dans cette même logique,l'enseigne Monoprix, dans le but de faire face à la concurrence de la nouvelle puissance de Carrefour, a opté pour le rachat des petits concurrents. Ainsi, Monoprix a intégré à son réseau la chaîne Le Passage en 2001 (9 magasins) et à la chaîne Touta en 2003 (11 magasins).Afin d'étendre son réseau de points de vente sur une grande partie du territoire national, Monoprix a procurétoutes les actions de l'entreprise « Tunisian Shopping Spaces » « Sahara Confort » localisés principalement au sud du pays.

Quant au magasin général pionnier de la grande distribution au pays, il a connu un développement composé. L'enseigne publique a été privatisée en 2007 après l'acquisitiondu capital détenu par le secteur public par les deux groupesBayahi et de Poulina. En automne de 2008, le Magasin général achète $35 \%$ des groupes Mabrouk et $34 \%$ des groupes Hamrouni dans l'entreprise BHM Promogro.En 2012, la société a signé un accord de partenariat avec Auchan qui prévoit une participation de $10 \%$ au capital de la société.Une année plus tard, le groupe a annoncé la fusion de ses trois enseignes de grandedistribution Magasin général, Promogro et Magro sous l'enseigne de MG. Toutes ces opérations de rachat et de fusions ont permis à l'enseigne Magasin général de s'imposer et de gagner une part assez importante deséchanges de la grande distribution. 
Le paysage de la grande distribution vient de s'enrichir en 2014 par une nouvelle enseigne « Aziza » (cartes 2 et 3). Entre 2014 et 2018, Azizaaaugmentéle nombre despoints de ventes à170 pour se diffuser sur une grande partie du territoire national. Cette nouvelle enseigne est détenue par le groupe tunisien multisectoriel privé« GROUPESLAMA », important conglomérat alimentaire. Ce dernier est actif dans l'agroalimentaire via les marques de la GIAS (Goldina, Nejma, Oleiva), Glaces (Nassim), Hightech (MB).

\section{LE HARD DISCOUNT EN TUNISIE : L'ENSEIGNE AZIZA}

\subsection{Historique du Maxidiscompte Dans le Monde}

L'émergence du hard discount remonte au début à 1917.Le premier magasin discount (non alimentaire) a été inventé parBernie Shulmanaux Etats-Unis. Cependant, l'apparition effective du hard-discount alimentaire n'était qu'après la deuxième guerre mondiale en Allemagne. « Le concept a été forgépar les frères Albrecht fondateurs de l'enseigne Aldi »(Guillo P., 2008). Vu la faiblesse des « moyens financiers et les restrictions alimentaires, les frères Albrecht proposaient un assortiment limité de produits alimentaires sans marques à des prix très bas. Le succès de la formule repose sur 1'application stricte d'un concept quasi-industriel : des conditions d'achat optimisées du fait des gros volumes, une organisation logistique rigoureuse et une chasse aux coûts cachés.

L'absence de vendeurs, la présentation sommaire, les horaires d'ouverture réduits et 1'absence de publicité permettent de réduire les coûts au strict minimum » (Guillo P., 2008). « Le nouveau concept a profité de l'entrée du pays dans une période de récession économique. S'appuyant sur une stratégie de bas prix pour une qualité jugée acceptable, le hard discount est parvenu à répondre parfaitement aux besoins des consommateurs qui voyaient leur pouvoir d'achat stagner ou diminuer » (Guillo P., 2008).

À la fin des années 1980, «les réseaux allemands de hard discount, en premier lieu Aldi et Lidl, ont investi le territoire français avant de s'étendre progressivement vers les autres pays européens » (Enrico C., 2003). En 2000, le discount alimentaire à assortiment limitée était présent dans tous les pays européens avec 72 enseignes exploitant 29747 magasins et une part de marché de 14,9\% des produits alimentaires. (Enrico C., 2003).

Plusieurs définitions ont été avancées pour saisir les spécificités de la nouvelle forme de distribution. Selon la terminologie retenue par l'INSEE, « un hard discount ou maxi discompte est un établissement de vente de produits alimentaires en libre-service sous enseigne spécifique d'une surface de vente de 400 à $800 \mathrm{~m}^{2}$ » (www.insee.fr). «L'assortiment propose est limité aux produits de base»(www.insee.fr).

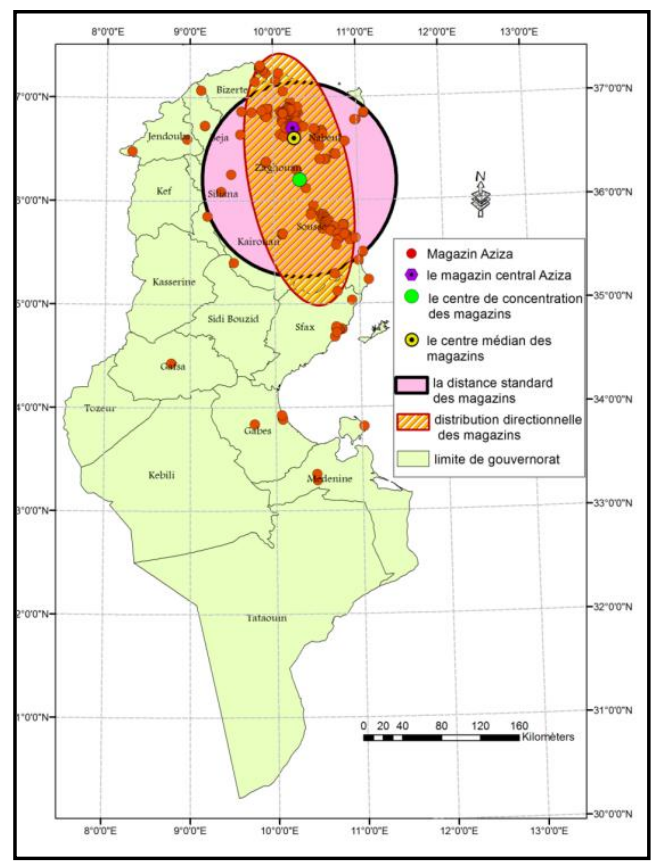

Carte2. Répartition spatiale des magasins Aziza en Tunisie en novembre 2018

Source des données : $w w w$.aziza.tn 
Des commentaires précisent que « ces magasins ne constituent pas une catégorie particulière dans les nomenclatures. Ils sont repérés par leurs enseignes et sont classés suivant leur superficie en superettes (moins d'un tiers d'entre eux) ou en supermarchés (Guillo P., 2008).

Selon la définition de Panorama/Points de vente, « un magasin de maxidiscompte est un libre-service alimentaire avec un personnel réduit, une présentation sommaire, un assortiment limité aux produits de base, des prix bas, peu ou pas de marques nationales, mais des produitssous marque de distributeur et des produits sans marque. Ces points de vente, qui sont parfois des locaux loués » (Guillo P., 2008).

En bref, le hard discount se distingue des autres formes de distribution par « le minimalisme en matière de services de vente et d'aménagement des magasins, et l'étroitesse relative de l'assortiment, concentré sur les produits de type marques de distributeurs (MDD) à bas prix » (http://www.cgedd. developpement-durable.gouv.fr)

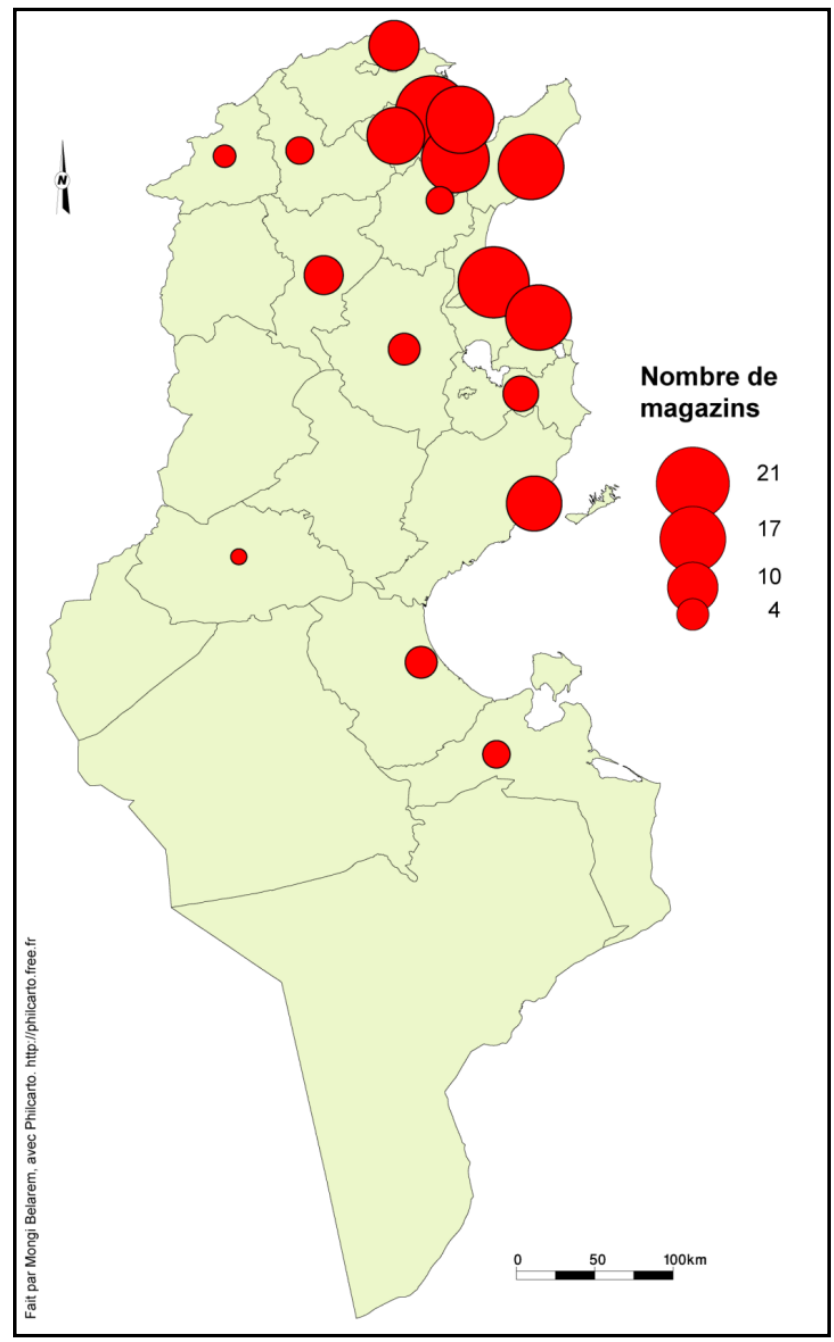

Carte3. Répartition spatiale des magasins Aziza en Tunisie par gouvernorat en novembre 2018

Source des données : $w w w$.aziza.tn

\subsection{L'enseigne Aziza : une Forme Hybride du Hard Discount}

En effet, la stratégie d'un distributeur se construit en premier lieu au point de vente : d'abord par la mise au point d'un concept magasin, ce qui signifie un aménagement, un assortiment, une formule de vente et donc, au total, une cible consommateur» (Lhermie C., 1996). D'ailleurs, le concept de magasin maxidiscompte reste marqué par ses caractéristiques d'origine dont les plus importantes sont l'exigüité de la taille des magasins, l'assortiment restreint et une recherche de la proximité au moindre coût. «Un magasin de maxidiscompte est souvent un établissement dédié à la commercialisation de biens alimentaires, ainsi que de produits d'hygiène et d'entretien, dont la surface de vente est bien 
souvent comprise entre 300 et $800 \mathrm{~m}^{2}$. » (https://www.troyes.cci.fr/). Le hard discount se distingue aussi par «l'absence d'investissement dans l'aménagement du magasin, la présentation des produits, l'emploi d'un personnel en nombre réduit » (http://pf-mh.uvt.rnu.tn). Ces facteurs représentent les clés de réussite des magasins hard discounts. En effet, «la réduction des frais du personnel et d'aménagement du magasin permet aux discounters de baisser les prix et d'accroitre les ventes » (https://www.troyes.cci.fr/). Avec « des magasins très spartiates aux services limités, tous les coûts de gestion et les investissements sont réduits au minimum. La forte rotation du capital permet d'obtenir, en dépit d'une faible marge brute, une bonne rentabilité » (Colla E., 2003).

L'enseigne «Aziza » n'a pas échappé au modèle général des magasins hard discount. Cette enseigne propose des magasins de 300 à $600 \mathrm{~m}^{2}$ de surface de vente, ouverts selon de larges amplitudes horaires (08h-22h) durant toute la semaine. Ces formats de points de vente ont été conçus pour s'adapter aux tissus denses. «Les marchandises sont présentées sur des gondoles dans les cartons d'origine (les articles permanents), alors que les produits en promotion sont exposés dans des tables de fouilles ou sur des palettes. L'assortiment est très restreint : en moyenne, 600 références». (Https://pastel.archives-ouvertes.fr/) (Photos 1, 2 et 3).

L'environnement de vente se caractérise aussi par un personnel réduit composé essentiellement d'un responsable et 6 commerciaux. Le responsable du magasin est chargé de lancer les commandes des achats et de la gestion des stocks, ainsi qu'à l'encadrement de l'équipe. Cependant, le commercial polyvalent est chargé à la fois d'effectuer les encaissements auprès des clientset s'occuper de tous les aspects de la relation marchande (composition des assortiments, aménagement, mise en place des produits...).

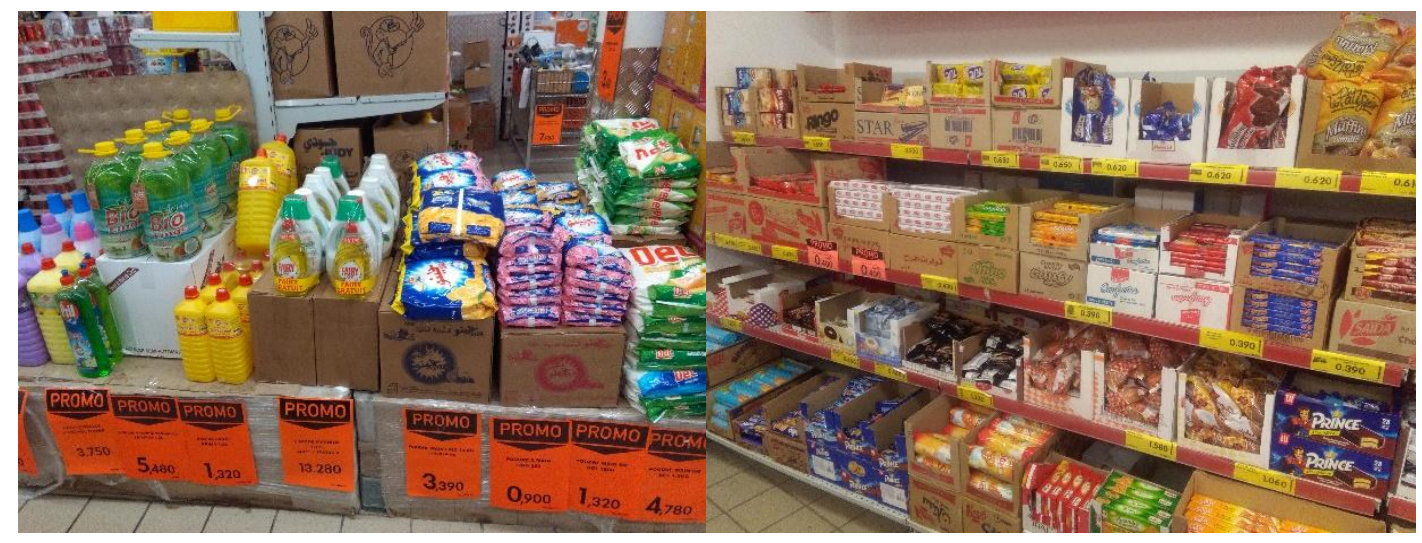

Photo1. Articles en promotion sur palette Photo2. Articles permanents sur gondole

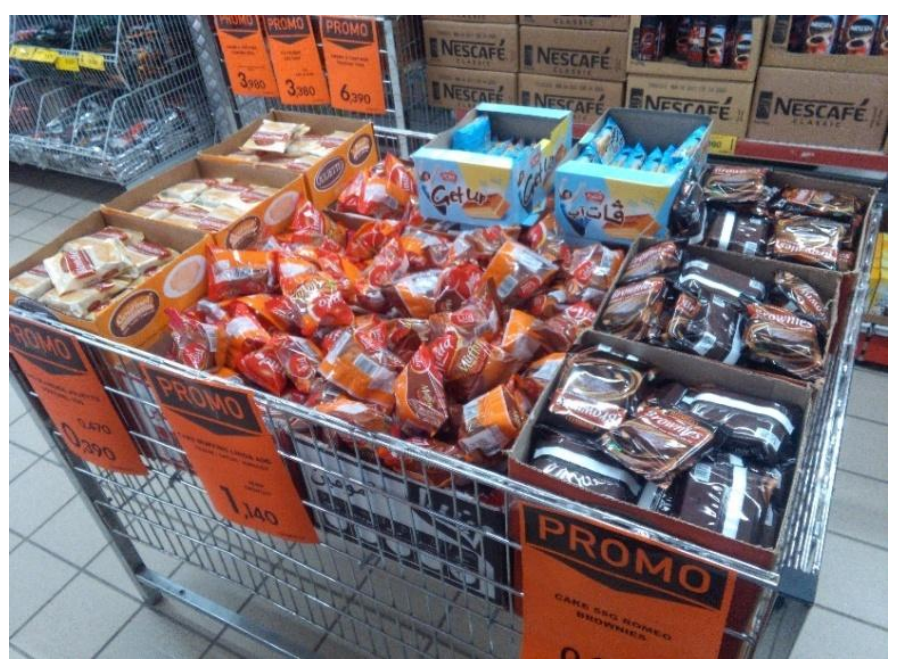

Photo3. Articles en promotion sur table de fouille 


\subsubsection{Les bas prix}

Le concept hard discount repose essentiellement sur des prix bas et un assortiment limité. Dans les pays européens, ces magasins ont été pour longtemps considérés comme le marché des pauvres. Les hard discounters, via leurs prix bas et leur assortiment restreint, séduisent une clientèle de plus en plus large. Cette formule apportera une réponse adéquate surtout aux demandes des clients à faible pouvoir d'achat. Ces dernierssont de plus en plus capables de recontrôler leurs dépenses et de réduire la facture globale de leurs courses.

Cette politique des prix bas a permis aux maxidiscompteurs de s'imposer dans le domaine de la grande distribution et s'emparer d'une part du marché. Toutefois, pour « consolider cet avantage concurrentiel centré sur la domination par le bas prix, les maxidiscompteurs ont adopté aussi une stratégie de différentiation qui contribue à la maîtrise des coûts. Pour cela, les maxidiscompteurs s'appuient sur des marques exclusives, inconnues du public, qu'ils créent eux-mêmes et qu'ils assemblent pour définir leur offre. Ils cherchent ainsi à fidéliser leur clientèle en y associant l'image de leur enseigne. Ces marques exclusives jouissent d'un positionnement très compétitif par leur qualité. Leur prix est nettement inférieur à celui des marques nationales grâce à des volumes d'achat très élevés, aux rapports particuliers entre producteurs et distributeurs et à certaines économies réalisées au cours de la chaine de l'offre, en particulier en termes de logistique $\gg($ Guillo P., 2008).

L'enseigne «Aziza »a accordé ce facteur une grande importance. Elle s'est efforcée, dès son démarrage, àfournir les prix «toujours les moins chers ». Pour atteindre cet objectif de baisse de prix, la nouvelle enseigne, à l'image de la plupart des discounters dans le monde offrant les « Marques De Distributeurs » (MDD) comme alternatives aux marques connues souvent plus chères,s'est engagée dans l'amplification de ses marques. Dansun contexte d'incertitude politique et économique, qui a eu une répercussion véritablement négative sur le pouvoir d'achat du consommateur tunisien, la nouvelle enseigne a voulu jouer la carte du discount et des prix cassés, pour relancer la consommation des ménages.Sur son site officiel, l'enseigne promet d'offrir des produits de marque aux meilleurs prix en limitant les charges et les dépenses inutiles.

Les MDD, proposés par l'enseigne «Aziza »,couvrent des catégories de produits à forte rotation en particulier la majorité des produits alimentaires (El Béhi, Nassiha, Omnia, Complices, Cœur d'or...) et quelques produits de nettoyage et détergents (ivi).

L'implication croissante de l'enseigne «Aziza» dans la conception et la production de ses propres marques l'a incité à établir des partenariats avec des petits PME, mais aussi par les innovants de marques nationales qui sont généralementcompétitricesdes MDD. Couramment, les produits MDD sont des produits copiés aux produits de marques nationales ou internationales avec quelques modifications dans la composition. "L'absence de frais de commercialisation (vente, marketing, merchandising, publicité, promotion) pour ces produits offre une plus grande marge bénéficiaire pour les distributeurs » (https://pastel.archives-ouvertes.fr/).

Les MDD de «Aziza» séduisent de plus en plus les clients grâce à leurs prix compétitifs. Cependant,l'évolution de la part de ces marques dans les ventes demeure largement tributaire de la confiance que les clients leur attribuent. Ainsi, «si le client est satisfait du rapport qualité/prix d'une marque de distributeur, il tendra à renouveler l'acte d'achat et à être captif puisqu'il ne retrouvera pas ce produit chez les concurrents. Plus la marque est reconnaissable pour son appartenance à l'enseigne, plus le phénomène s'accentuera. Dans ce cas, le distributeur dispose de deux atouts qui lui assurent un réel avantage concurrentiel : la fidélité à la fois à sa marque et à son point de vente. La fidélité est donc avant tout liée au prix ou plus précisément au rapport qualité/prix considéré comme acceptable en ce qui concerne la qualité et comme imbattable en ce qui concerne le prix » (Guillo P., 2008).Il est à noter à ce propos que les clients des magasins « Aziza »optentencore pour les grandes marques nationales, dans l'chat de plusieurs articles.

Certes, l'enseigne« Aziza » n'a pas échappé à la règle générale des discounters pour les prix cassés. En effet, elleoffreles prix les moins chers du marché (généralement ils sont de $-20 \%$ par rapport aux prix de vente des autres enseignes).La réduction des prix pour les marques nationales provient de l'achat en grande quantité des produits surtout lorsqu'ils sont proposés en vente promotionnelle. L'augmentation de la taille de l'enseigne lui a permis de renforcer son pouvoir de négociation avec ses fournisseurs.L'enseigne «Aziza» est devenue un revendeur incontournable pour plusieurs fournisseurs. 
Au départ, la campagne publicitaire de«Aziza»s'appuyait essentiellement sur ses catalogues hebdomadaires distribués dans les magasins présentant les produits en promotion de la semaine. Pour accélérer la diffusion de son image de discompteur«Aziza» a même fait l'objet de spots publicitaires dans une chaîne télévisée privée.Cependant son site internet propose uniquement leurs offres promotionnelles du moment, les adresses des magasins et quelques offres d'emploi.

\subsubsection{L'optimisation des flux logistiques : un point déterminant}

Depuis des décennies, «la fonction logistique est devenue une source d'avantage concurrentiel dans la grande distribution.Avecl'extension des réseaux de leurs points de vente,les groupes de distribution sont de plus en plus confrontés à l'alourdissementdes coûts de la logistique et du traitement administratif des achats. Dès lors les distributeurs vont investir les activités logistiques qui jusque-là étaient prises en charge par les producteurs » Senkel M., (2005). « Depuis le début des années 1980,à une organisation logistique maîtrisée par les producteurs s'est substituée au fil du temps une organisation largement prise en charge par les firmes de grande distribution » (Senkel M., 2005).

Selon Senkel M. (2005), « la maîtrise de la logistique permet aux distributeurs de répondre à un triple objectif : augmenter leur rentabilité, accroître leur flexibilité et améliorer le service à la clientèle de plus en plus versatile à travers la limitation des ruptures de stocks, par la capacité à traiter l'augmentation du nombre de références et la plus forte rotation des produits ou des collections et par l'amélioration de la réactivité face aux nouvelles tendances du marché ».Afin d'atteindre leur triple objectif (coût, flexibilité et service), les entreprises de distribution se sont équipées d'outils logistiques : entrepôts, plates-formes et moyens de transport. Ces outils leur permettent de centraliser les flux d'approvisionnement venant de nombreux fournisseurs sur un nombre restreint de sites, d'y effectuer des opérations de préparation de la marchandise comme le conditionnement, l'étiquetage... avant de livrer quotidiennement les magasins de détail(Senkel M., 2005).

Consciente de cet enjeu majeur que représente la maitrise de la fonction logistique et des gains qu'elle en pouvait retirer,la nouvelle enseigne de distribution Aziza amis en place des infrastructures et des procédures logistiques à l'attention de son réseau de points de vente. Pour assurer la logistique au quotidien et l'approvisionnement de ses entrepôts et ses points de vente, l'enseigne Azizaa fait le choix de l'externalisation complète de ses moyens logistiques à la société «Lumière Logistique » filiale logistique du groupe Slama. Créée en 2012, lasociété avait pour objectifde récupérer les activités logistiques communes aux différentes entités du Groupe. Ce dernier a réussi à travers la création de «lumière logistique » d'accaparer une bonne part du marché « des activités de prestations de services logistiques à destination des métiers des produits de grande consommation « FMCG » et de la distribution »(http://webradar.me/). En effet, la société «lumière logistique » propose ses services à toutes les entreprises du domaine avec une offre comprenant une expertise des métiers du transit, du transport, de l'entreposage, du conditionnement, de la distribution et du service à la clientèle » (http://webradar.me/).La société compte 10 entrepôtslocalisés en majorité sur le Grand Tunis (Bou Argoub, El Mghira, BirElKassaa...)et85 véhicules au départ.

Avec la multiplication du nombre de ses magasins, l'enseigne Aziza distributeurs a été amené à créer de nouveaux entrepôts. Elle a opté pour une organisation basée sur in entrepôt principal. Ce centre de distribution a été inauguré, en Mars 2015, dans la localité de Sahline dans le gouvernorat de Monastir.

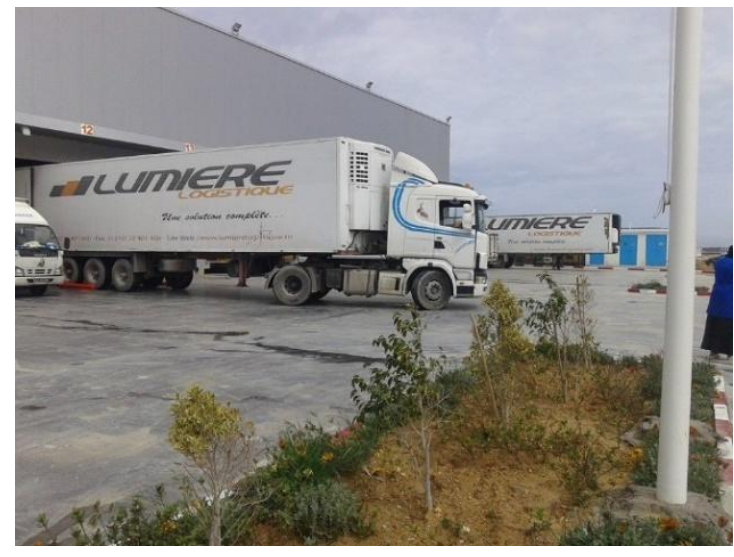

Photo4. Dépôt Sahline 
D'autres dépôts régionaux ont été implantés (Sfax) qui reçoivent les produits de l'entrepôt principalde Sahline(photo 4) ou directement des producteurs pour les transférer aux nouveaux magasins. «L'organisation logistique de l'enseigne correspond au modèle logistique 2, celui des livraisons des usines vers les centres de distribution (CD) des grands distributeurs, ceux-ci étant ensuite chargés d'approvisionner leurs magasins. Les CD sont gérés directement par les distributeurs »(Chanut O., Paché G., 2012).«Ce modèle logistiques'appuyant sur la mutualisationdes stocks d'un même grand distributeur dans un ou plusieurs $C D$ et de réduire d'autant les réserves en magasin. Ces dernières sont progressivement transformées en surfaces de vente »(Chanut O., Paché G., 2012).Dans l'objectif de la réduction les coûts de transport et de stockage, l'enseigne a opté pour l'optimisation deschargements des camions (taux de remplissage) impliquantla réductiondes tournées d'approvisionnements. «Souvent, les tournées à partir de l'entrepôt, sont fondées sur le groupage des produits à destination des différents magasins » (https://hal.archives-ouvertes.fr/hal-01767220).

En effet, « la maîtrise des infrastructures logistiques associée à la centralisation des activités d'achat a permis à l'enseigne Aziza de faire des économies d'échelle. Cette autonomie au niveau logistiqueainsi que les achats groupés pour l'ensemble des magasins autorisent une puissance d'achat et un pouvoir de négociation accrus face aux fournisseurs » (Chanut O., Paché G., 2012).

En outre, la maîtrise de la fonction logistique de la part de l'enseigne «Aziza» lui a permis aussi d'anticiper et de veiller à toutes sortes de ruptures aux niveaux des stocks surtout lors des opérations de promotions.Par une mainmise sur les opérations logistiques, on peut dire que la nouvelle enseigne a réussi à assurer d'une manière régulière l'approvisionnement des points de vente, et de prévoir toute défaillance logistique pouvant atténuer la portée des actions marketing et commerciales envisagées.

Cependant, la gestion d'organisation logistique ne peut pas se limiter à la seule considération du flux physique. Elle nécessite en outre, une maîtrise des flux d'informations. "Cette maîtrise des flux caractérisant la majorité des canaux de distribution moderne provient du développement des systèmes d'informations. Cantonné en prémisse autour de l'unité physique du produit, le canal de distribution, tend à s'organiser progressivement autour de l'information relative au produit : information sur les ventes passées et les prévisions de ventes à court terme, sur l'état des stocks dans le canal, et sur les capacités productives des fabricants » (Filser M., 1998).

S'inspirant des expériences de grands distributeurs en matière de logistique, l'enseigne « Aziza »a tenté dès le début de contrôler les flux d'informations. L'enseignea investi dans l'informatisation des magasins et dans des moyens automatisés de transfert et de traitement informatique des données en interne permettant de consulter, en temps réel, les stocks disponibles. Pour optimiser la gestion de ses stocks, du back-office, de la mobilité en magasin et du merchandising, l'enseigne a choisi de coopérer avec le fournisseur mondial leader deplateformes décisionnelles SymphonyEYC en adoptant la solution Gold solution logicielle pour la distribution. Selon l'enseigne, le choix deSymphony EYC est justifié par la facilité de paramétrage de sa solution et de sa connaissance du marché de la distribution nationale, vu qu'il a déjà accompagné le développement de Monoprix et Casino (Groupe Mabrouk).

\section{Strategie D'implantation des MAgasins AZIZA :LA PROXIMITE COMME SOURCE DE DIFFERENCIATION}

\subsection{Répartition Spatiale sur le Territoire National}

L'enseigne Aziza a retenu notre attention car elle présente un intérêt pour la dynamique de sa stratégie d'expansion au cours des quatre dernières années.La chaîne a ouvert ses premiers magasins en 2014 pour atteindre une centaine de points de vente à la fin de 2017.Dès lors,les ouvertures s'accélèrent. L'enseigne prévoit atteindre le nombre de 200 magasins à la fin de 2018, et 300 à la fin de l'année 2019.La stratégie d'implantation de l'enseigne « Aziza » était efficace mais coûteuse, car elle exige de forts investissements avec l'accélération du rythme des ouvertures. Cette nouvelle exigence a poussé l'enseigne à coopérer avec le groupe d'investissement émirati «Abraj ». Cettecoopération lui a permis de développer son réseau de magasins.

«Au niveau de la répartition spatiale, à l'image des principaux opérateurs de la grande distribution en Tunisie, l'enseigne Aziza semble avoir prioritairement investi la frange littorale notamment le Grand Tunis. La grande distribution moderne reste ainsi concentrée dans les grandes villes littorales du pays 
où les habitants jouissent d'un niveau de vie qui leur permet d'accéder à la sphère de consommation »(https://hal.archives-ouvertes.fr/hal-01767220).La plupart des magasins Aziza sont implantées actuellement que dans les principales villes alors que les régions intérieures n'ont pas encore été suffisamment investies en particulier par les grandes surfaces. À titre d'exemple, sur 177 magasins à l'échelle nationale,70 sont localisés sur le Grand Tunis. L'expansion de la nouvelle chaîne s'est concentrée aussi autour des grandes villes du sahel (Sousse, Monastir, Mahdia)qui regroupent 42 magasins.Après la consolidation de son ancrage dans la région du Sahel, l'enseigne a commencé à essaimer ses premiers magasins dans le gouvernorat de Sfax (cartes 7, 8 et 9).

L'examen des cartes 2 et 3permet de constater que la stratégie de couverture de l'enseigne Aziza se caractérise parune avancée progressive vers les gouvernorats de l'intérieur notamment ceux du Nordouest et la poursuite d'une densification dans les territoires conquis, particulièrement le Grand Tunis et les gouvernorats du Sahel (Sousse, Mahdia et Monastir).La stratégie d'expansion de l'enseigne semblesuivre une diffusion hiérarchique qui consiste à s'implanter,avant tout dans les grandes pôles et par la suite dans les petites selon la théorie des places centrales.

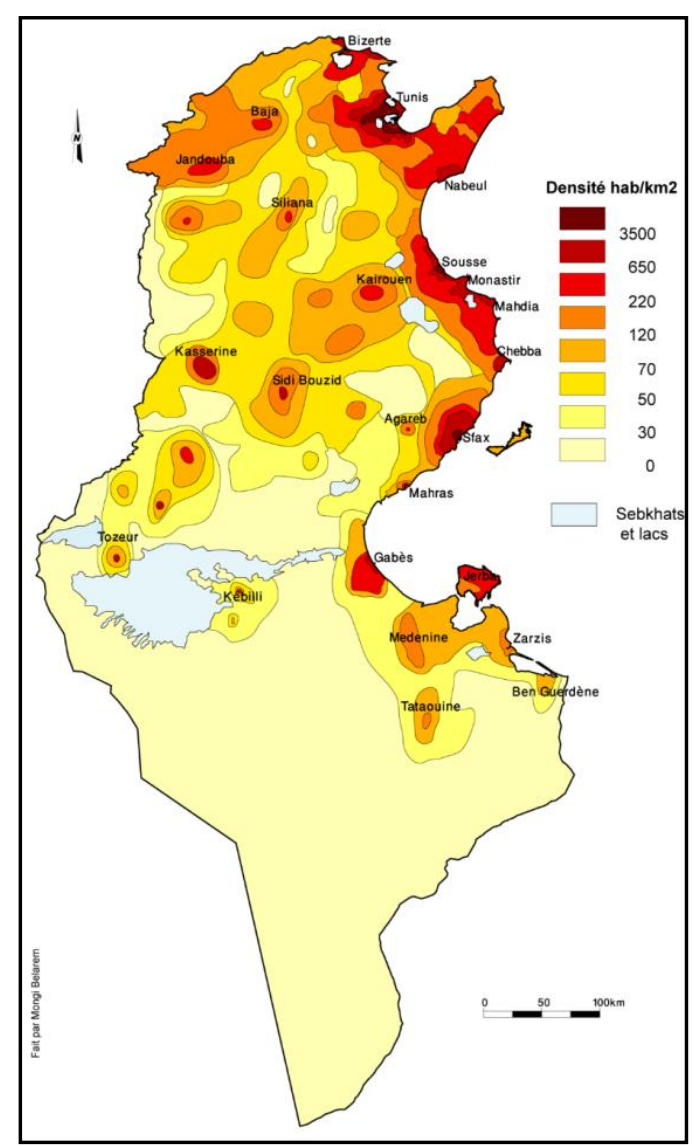

Carte4. Répartition spatiale des densités de la population Tunisie en 2014

Source de données : www.ins.tn

Cette conquête progressive du territoire procède à une expansion sur le territoire par l'effet de grappe. Fréquemment utilisé par les enseignes de maxidiscompte, l'effet de grappe consiste à implanter simultanément plusieurs points de vente à proximité d'une base logistique. Cette stratégie s'avère efficiente. Mais elle exige, outre de faibles contraintes en matière d'implantation, un marché ouvert et peu concurrentiel(Guillo P., 2008).

Cette accélération de l'expansion des magasins «Aziza » s'inscrit dans la stratégie territoriale des distributeurs à bas prix. En effet, «pour préserver l'avantage concurrentiel dont jouit le format, ces distributeurs doivent éviter des ruptures dans le processus de diffusion de 1'enseigne. Pour cela, face à la concurrence, ils sont tenus d'intensifier leur couverture spatiale en accélérant la vitesse de propagation du front de diffusion tout en recherchant la cohérence dans les localisations. Cette préoccupation est au cœur des stratégies mises en œuvre par toutes les enseignes pour la conquête de nouveaux marchés » (Guillo P., 2008). 
La carte de la répartition géographique des magasins «Aziza » est presque calquée sur celle de la population et à celle de la densité de la population. Les cartes 2, 3, 4 et 5illustrent bien des disparités entre les régions littorales très urbanisés et peuplés captant la majeure partie des implantations et les régions intérieures moins peuplés. L'observation de ces dernières cartes montre clairement ce clivage littoral/intérieur.

Rapportées au nombre d'habitants, la densité des magasins «Aziza » oscille entre 1 magasin pour 25430 habitants au gouvernorat d'Ariana et 1 magasin pour 347500 habitants à Gafsa (carte 7). Les quelques gouvernorats du Nord (Bizerte, Nabeul et Ben Arous) bénéficient des ratios supérieurs à la moyenne nationale. Le sous équipement en magasins «Aziza » s'aggravait avec l'éloignement des villes du littoral. L'enseigne n'a pas encore opéré dans 3 gouvernorats du sud : Tataouine, Tozeur et Kébili n'ont pas encore.

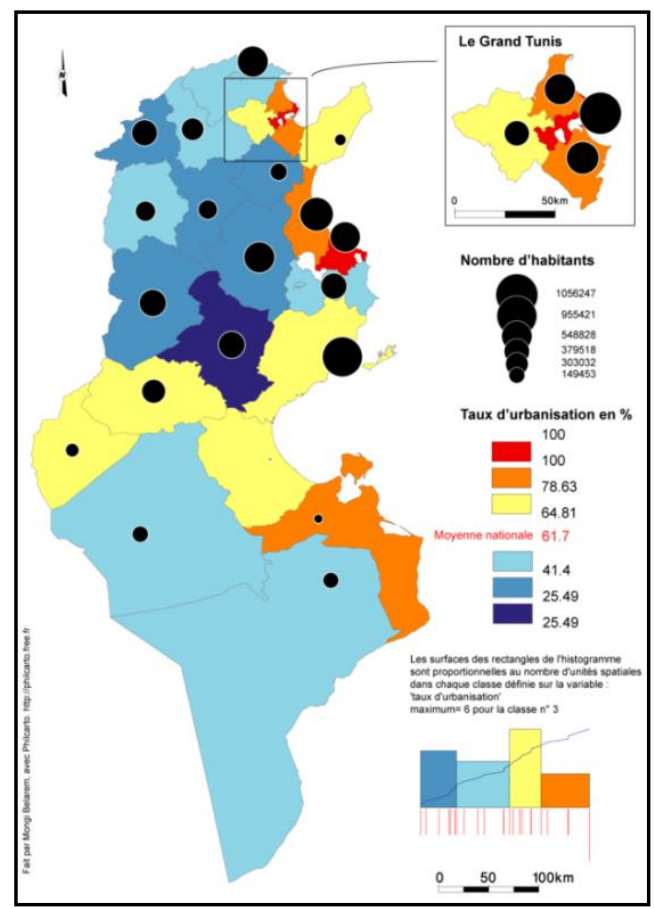

Carte5. Répartition de la population et des taux d'urbanisation en Tunisie en 2018

Source de données : $w w w$.ins.tn

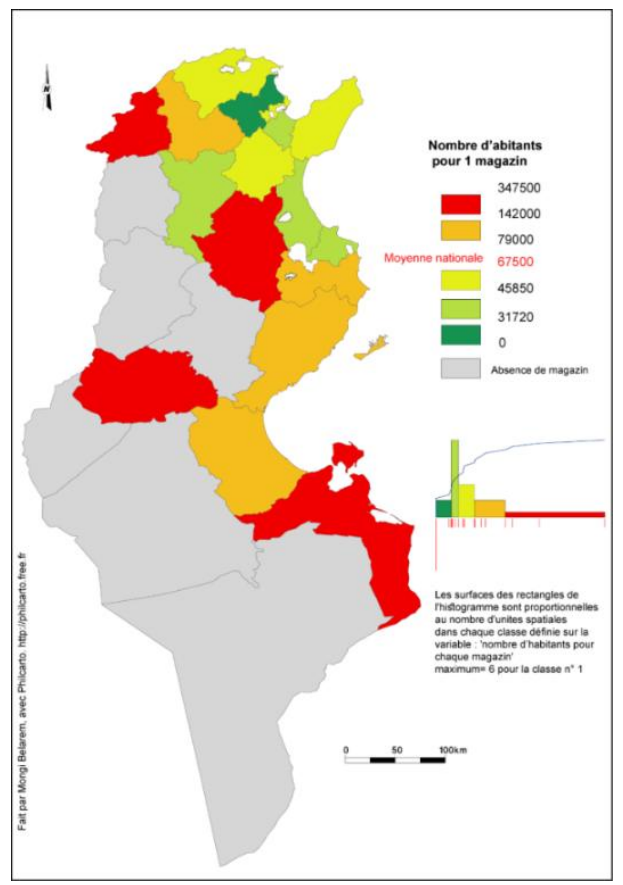

Carte6. Nombre d'habitants pour chaque magasin Aziza par gouvernorat en novembre 2018

Source des données : $w w w$.aziza.tn et $w w w . i n s . t n$ 
A l'échelle nationale, la densité actuelle des magasins «Aziza» est relativement faible. Elle est de l'ordre d'un magasin pour 67500 habitants.Le gouvernorat d'Ariana détient la première place du palmarès national avec une densité de l'ordrede 25430. II faut cependant noter la disparité entre les régions littorales. Par exemple, dans la région du Sahel, les chiffres révèlent un décalage en termes d'équipements et d'implantation de magasins «Aziza» entre Sousse et Mahdia. Ce dernier gouvernorat enregistre une densité de 1 magasin pour 78999 habitants alors que celui de Sousse bénéficie d'une densité de 1 magasin pour 32437 habitants.

\subsection{La Proximité et le Petit Commerce Alimentaire}

En Tunisie, le commerce alimentaire de proximité était figé pour longtemps dans des structures traditionnelles.Ces structures comportaient aussi les souks, les marchés municipaux, les vendeurs ambulants. L'offre de proximité était réduite à la distribution des produits de première nécessité, intégrée à l'habitat, hiérarchisée et fréquentée à pied. Depuis des décennies, la petite épicerie, demeure au cœur des pratiques commerciales quotidiennes dans les quartiers résidentiels. Le petit commerce alimentaire, au sein des quartiers des villes tunisiennes, s'inscrit dans un rapport de proximité incontournable.En effet, «la proximité implique un rapport de distance et de temps privilégié envers un espace ou un équipement dont on est proche, que l'on est amené à fréquenter régulièrement et quasi quotidiennement. L'espace de proximité se confond alors avec celui de l'interconnaissance, l'espace que l'on connaît, où l'on est connu et reconnu »(Gasnier A., Péron R., 2000).

Le commerce alimentaire de proximité était exercé dans de petits magasins ne dépassant pas souvent $20 \mathrm{~m}^{2}$. Depuis le début des années quatre-vingt-dix, on a observél'apparition des superettes. La majorité de ces superettes est le résultat d'une transformation endogène de l'épicerie lorsque les conditions la permettaient(Bennasr A., Azouazi Th., 2010).

Les épiceries servent une clientèle régulière peu mobile et captive, à faible pouvoir d'achat et contraint de s'approvisionner en petite quantité. Malgré son assortiment restreint et ses prix relativement chers, l'épicerie du quartier jouit d'unefréquentation soutenue (rythmes quotidiens ou pluri-hebdomadaires). La majorité des habitants des quartiers s'adressent régulièrement aux épiceriesprofitant ainsi d'un service de proximité à la fois spatiale et temporelle, grâce à leurs localisations en plein quartier et leurs horaires d'ouverture très larges.

L'épicerie a représenté pour longtemps la forme dominante voire exclusive de l'offre commerciale dans les quartiers notamment populaires. Cette domination procède essentiellement de «la proximité géographique et psychologique, la vente à crédit et en vrac en petites quantités et la souplesse des heures de travail qui constituent des atouts privilégiant le petit commerce. Mais, il n'en reste pas moins que plusieurs entraves limitent son développement en l'occurrence la faible capacité de financement et de modernisation, le manque de communication, la logistique rudimentaire et le faible niveau d'instruction des petits commerçants » (Ben Hassen S., 2008).

En dépit de son recul incontestable à l'échelle mondiale,le commerce alimentaire reste prépondérant en Tunisie en raison de plusieurs facteursdont la plus importante le faible équipement des quartiers en moyennes et grandes surfaces. Partant de constat, la nouvelle enseigne «Aziza » est partie pour la reconquête des différents types quartiers (aisés et populaires) en développant des petits et moyens formats de magasins à proximité des habitants.

\subsection{Aziza et la Proximité}

Le contexte national et celui international ont influencé la stratégie de «proximité » de la grande distribution. Ainsi, à l'échelle internationale, les distributeurs se sont retrouvés, à l'aube du XXI ${ }^{\text {ème }}$ siècle, plus que jamais confrontés à la saturation économique de leurs grands formats(Lestrade $\mathrm{S}$., 2013). À l'échelle nationale, les opérateurs de la grande distribution ont investi « la proximité » pour gagner de nouveaux parts du marché.En effet, plusieurs enseignes ont tenté d'investir la proximité. Rappelons à titre d'exemple, la stratégie du Magasin Général à partir de 2011 et qui consiste à implanter des supermarchés de plus de $500 \mathrm{~m}^{2}$ (mg proxi), situés à proximité de votre quartier. Cependant, ces magasins ont été sélectionnés pour attirer une clientèle relativement aisée et à fort pouvoir d'achat. 
Partant de constat, l'enseigne «Aziza » a fondé sa stratégie d'implantation au niveau local. L'attention de la nouvelle enseigne est attirée par ce potentiel de clientèle captive habitant notamment les quartiers populaires. Elle a vu en ces nouvelles destinations de véritables opportunités de développement. Il s'agit en effet de territoires relativement « vierges » à l'écart de toute concurrence ou de nombreux besoins restent à satisfaire. S'appuyant sur ses bas prix, l'enseigne « Aziza » décide de s'attaquer aux clientèles plus modestes des quartiers populaires.

Depuis 2014, la nouvelle chaîne de distribution Aziza affirmeson intérêt au développement d'un commerce « de proximité »: C'est du moins ce qui ressort desslogans avancéspar le groupe sur son site officiel concernant sa nouvelle formule de magasins(https://www.aziza.tn).

L'opportunité d'implantation de l'enseigne Aziza dans les quartiers populaires et les zones à forte densité tient à leur faible équipement commercial qui se limitait souvent aux petits commerces alimentaires (épiciers) et à l'absence de concurrents de grande distribution dans ces zones. La nouvelle chaîne est partie ainsi à la conquête d'une clientèle encore relativement peu exploitée en Tunisie. Avec la multiplication de ses points de vente dans les quartiers, surtout populaires, de différentes villes dans la majorité desgouvernorats, elle tente d'accaparer de nouveaux parts de marché.

La réduction de la taille moyenne des points de vente a favorisé leur intégration dans les zones résidentielles à forte densité. Pour l'implantation de nouveaux magasins, l'enseigne «Aziza » a privilégié le voisinage des marchés et des commerces des quartiers déjà existants (boulangerie, boucherie, café, souk hebdomadaire...)bénéficiant de leur attractivité sur la population résidente.L'implantation des magasins dans ces quartiers est favorisée aussi par l'abordabilité des prix du loyer.

L'implantation des magasins «Aziza » au cœur des quartiers populairesparticipe «àla redynamisation d'anciens noyaux commerciaux. Ces équipements s'implantent en règle générale à proximité immédiate de l'ancien centre commerçant du quartier et deviennent de nouvelles locomotives attractives jouant à la fois sur la complémentarité entre formules commerciales et sur les prix bas » (Gasnier A., Péron R., 2000).

Cependant, pour l'enseigne «Aziza», la décision stratégique de s'implanter dans les quartiers populaires, si elle recèle des opportunités (terrain vierge), elle impose des contraintes.Azizaest contrainte de s'adapter aux spécificités de ce nouvel marché (les quartiers populaires). Ces derniers présentent la particularité d'une forte proportion de clients à faible pouvoir d'achat et à mobilité réduite. En effet, la contrainte budgétaire semble être un fort déterminant dans le choix des lieux d'achat chez les habitants quartiers populaires. Pour attirer et fidéliser cettenouvelle clientèle, l'enseigne «Aziza » a joué la carte des offres promotionnelles hebdomadaires et de la gratuité qui permettent aux yeux clients de faire des économies. Une attention particulière est portée à la clientèle féminine (les femmes souvent au foyer),en proposant une offre promotionnelle des ustensiles et accessoires de cuisine. Cette offre loin de répondre à des besoins spécifiques de consommation, a réussi à fidéliser cette clientèle.En outre, l'entendue des horaires et des jours d'ouverture de ces magasins favorise l'étalement dans le temps de leur consommation.

Un examen attentif des cartes de répartition des magasins dans le Grand Sfax (carte 7), deuxième agglomération du pays, illustre que l'enseigne «Aziza» a investi les quartiers populaires à forte densité (carte 8). En effet, la majorité de magasins sont implantés dans la zone péricentrale ainsi que sur les axes littoraux, concentrant les plus importants quartiers de la ville. A titre d'exemple, l'enseigne a implanté son premier magasin dans la zone de « r'bat», quartier populaire enregistrant la plus forte densité de la ville (plus de 20000habitants $/ \mathrm{km}^{2}$ ), (Ben Fguira S. et Belarem M.,2018). Deux autres magasins, ont été installés dans zone saltnia- Sidi Mansour, à proximité de Hay Bourguiba, l'un des grands quartiers d'habitat social (carte 9). Des magasins sont en cours de réalisation dans d'autres quartiers populaires de la ville (Cité Bahri et Cité Habib au sud) 


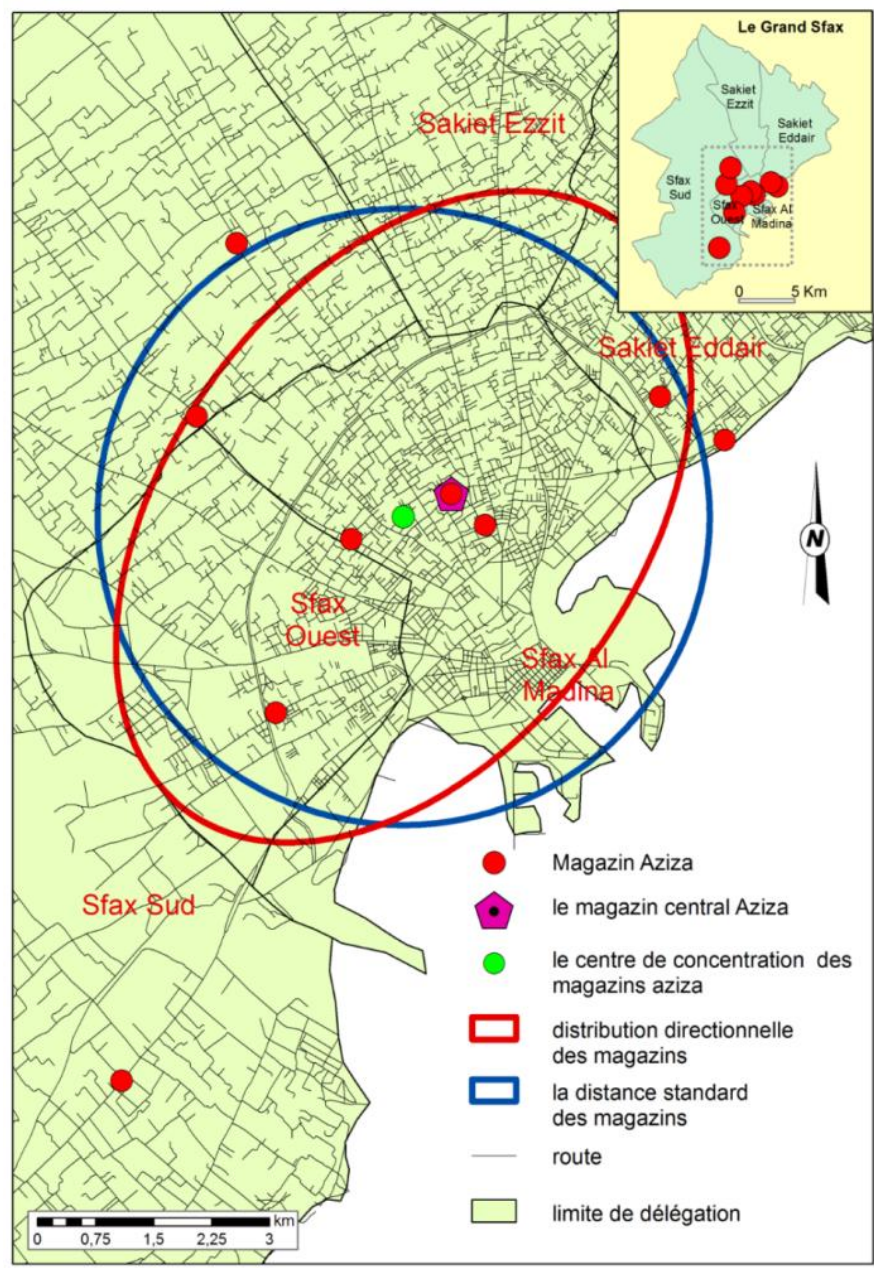

Carte7. Répartition spatiale des magazins Aziza dans le Grand Sfax en novembre 2018

Source des données : www.aziza.tn

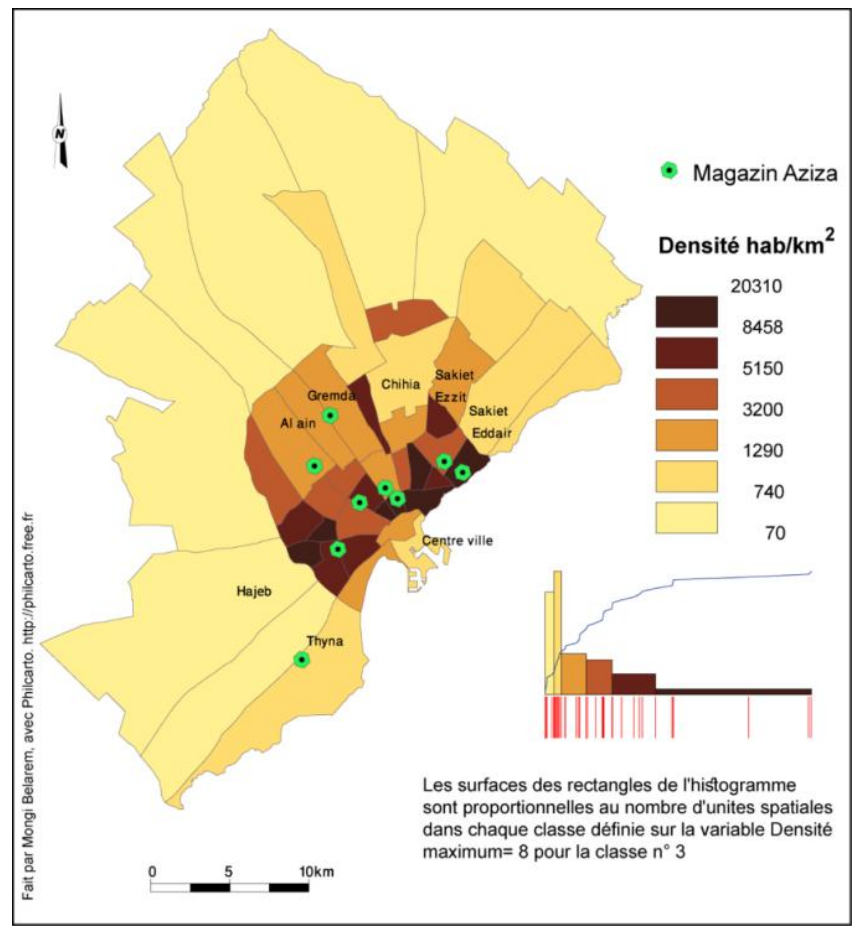

Carte8. Densité de la population par secteur et les magasins Aziza dans le Grand Sfax en novembre 2018

Source des données : $w w w . a z i z a . t n$ et $w w w . i n s . t n$ 


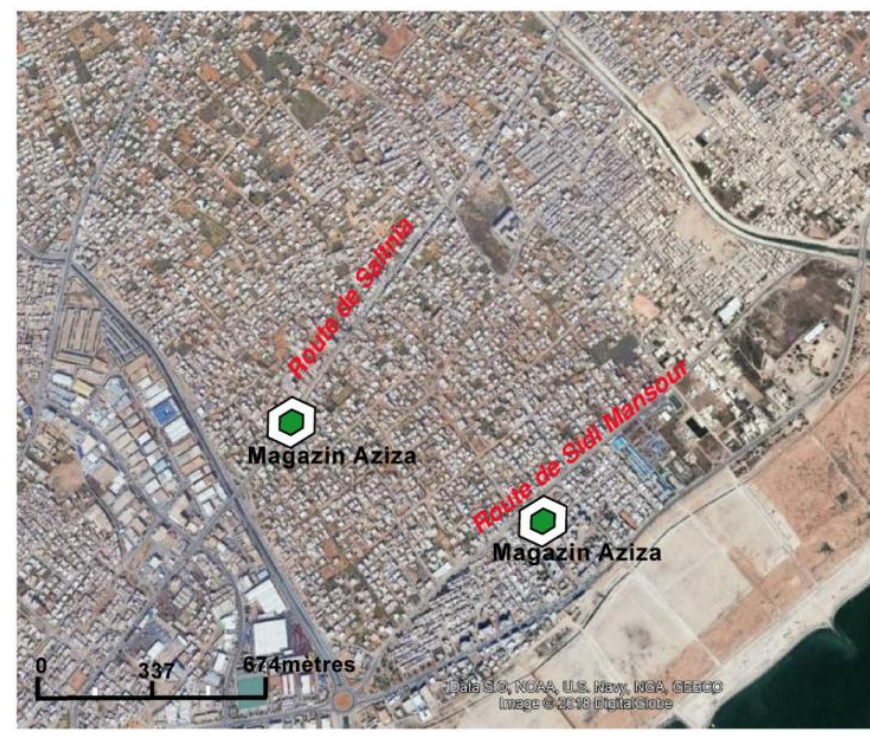

Carte9. Localisation des deux magasins de route Sidi Mansour et Saltnia à Sfax

Source des données : www.aziza.tn et https://earth.google.com

\section{CONCLUSiON}

Dans un contexte de crise économique dans la période postrévolutionnaire caractérisée par une chute du pouvoir d'achat et une inflation des prix, une nouvelle enseigne sous le nom de «Aziza » a émergé sur le marché tunisien de la grande distribution. À l'encontre des stratégies des anciennes enseignes de grande distribution installées dans le pays, «Aziza» a choisi d'adopter la nouvelle formule du «hard discount». La nouvelle enseigne a réussi partiellement à s'adapterà cette conjoncture économique et à étendre rapidement son réseau de magasins. De plus, elle est parvenue à attirer une bonne partie de consommateurs cherchant à réduire les dépenses de leurs coursesen choisissant la logiquedu hard discount et des prix les plus bas déployée par «Aziza ». Outre la logique du hard discount,la stratégie de l'enseigne "Aziza » s'est distinguée par le développement rapide de son réseau de magasins. La stratégie d'expansion repose particulièrement sur l'investissement de la proximité bénéficiant de nouveaux terrains inexplorés par les autres enseignes de la grande distribution particulièrementles quartiers résidentiels populairesdépourvus autrefois de ce type d'équipement commercial.

Par sa stratégie affichée du hard discount et des prix les plus sur le marché, la nouvelle enseigne a commencé à s'étendre et à grappiller des parts de marché du commerce alimentaire traditionnelet particulièrement des autres enseignes de la grande distribution. Contrairement aux anciennes formes de distribution en Tunisie, l'enseigne «Aziza » a cherché de s'implanter dans les territoires de proximité. Enréappropriant ces nouveaux terrains et en multipliant les points de vente à l'échelle nationale, la chaineest en traind'instaurer une sorte de barrière face aux actuels ou éventuelsconcurrents de la grande distribution.Afin d'améliorer sa performance et accompagner le développement de son réseau de magasins, qui avoisine aujourd'hui 400 unités, l'enseigne « Aziza » a fait associer d'autres actionnaires étrangers.

\section{REFERENCES}

Amine A., (2012), «La grande distribution dans les pays émergents : caractéristiques, enjeux et perspectives », Marché et organisations, $\mathrm{N}^{\circ} 15$, p. 117-141.

Ben Hassen S., (2008), Le petit commerce de l'horizon de 2020, Tunis, Université virtuelle de Tunis. 77 pages.

Ben Fguira S. et Belarem M., (2018) «Quel avenir pour le logement social en Tunisie ? », Confins [Online], $36 \mid 2018$, posto online no dia 30 junho 2018, consultado o 29 novembro 2018. URL : http://journals.openedition.org/confins/13450 ; DOI : 10.4000/confins.13450.

Bennasr A., (2005), «Les grandes surfaces à dominance alimentaire à Sfax », BouhouthJamiya, 2005, p.1-24.

Bennasr A., Azouazi Th., (2010), «Le commerce dans les politiques d'aménagement et de développement : le cas tunisien », Commerce et ville ou commerce sans la ville, p. 259-270.

Chanut O., Paché G., (2012), «Stratégies logistiques émergentes : de la grande distribution alimentaire aux réseaux contractuels », Marché et Organisations, $\mathrm{N}^{\circ} 15, \mathrm{p} .91-115$. 
Colla E., (2003), « L'expansion internationale du discount alimentaire », Décisions Marketing, N 30, p. 51-61.

Enrico colla, (2003), «l'expansion internationale du discount alimentaire », Décisions Marketing, $\mathrm{N}^{\circ}$ 8, p. 7581.

Filser M., (1998), «Évolutions et stratégies dans la distribution alimentaire : les apports des sciences de gestion », Économie rurale, $\mathrm{N}^{\circ} 245-246$, p. 9-15.

Gasnier A., Péron R., (2000), «Commerce et chalandise : la proximité éclatée », ESO Travaux et documents, $\mathrm{N}^{\circ}$ $14, \mathrm{p} .41-45$.

Guillo P., (2008), «Les stratégies d'expansion dans la distribution : le cas des maxidiscompteurs en France », Décisions Marketing, № 50, p. 7-18.

http://pf-mh.uvt.rnu.tn/

http://webradar.me

http://www.cgedd.developpement-durable.gouv.fr/IMG/pdf/01046801_rapport_cle2163e2.pdf

http://www.ins.tn

https://docplayer.fr/23985776-La-grande-distribution-au-maroc.html

https://earth.google.com

https://hal.archives-ouvertes.fr/hal-01767220/file/2012-MAORG-Chanut-Pach\%C3\%A9Strat\%20Log\% $20 \%$ C3\%A9mergentes.pdf

https://monoprix.tn

https://pastel.archives-ouvertes.fr/pastel-00994822/file/1996_EHEC_0043.pdf

https://ween.tn/fiche/mg-magasin-general

https://www.aziza.tn

https://www.carrefourtunisie.com

https://www.troyes.cci.fr/

Lestrade S., (2013), «Le commerce de proximité de la grande distribution en France : de nouveaux modèles de magasins pour de nouvelles relations avec la ville ? », Les Annales de la recherche urbaine, $\mathrm{N}^{\circ} 108$, p. 2435.

Lhermie C., (1996), «Les stratégies du distributeur », Décisions Marketing, N 8, p. 75-81.

Senkel M., (2005), «évolutions logistiques dans la grande distribution : de l'optimisation à la coordination des flux », Décisions Marketing, $N^{\circ} 39$, p. 67-78.

www.insee.fr

www.masca.com.tn

Citation: Sami Ben Fguira \& Mongi Belarem. "Le Commerce de Proximité de la Grande Distribution en Tunisie: Cas de la Chaîne Aziza" International Journal of Humanities Social Sciences and Education (IJHSSE), vol 8, no. 10, 2021, pp. 53-68. doi: https://doi.org/10.20431/2349-0381.0801007.

Copyright: () 2021 Authors. This is an open-access article distributed under the terms of the Creative Commons Attribution License, which permits unrestricted use, distribution, and reproduction in any medium, provided the original author and source are credited. 